\title{
Swift observations of the SFXT SAX J1818.6-1703 in outburst
}

\author{
P. Romano*, L. Sidoli ${ }^{\dagger}$, P. Esposito ${ }^{\dagger}$, V. La Parola*, J.A. Kennea**, \\ H.A. Krimm ${ }^{\ddagger}$, , M.M. Chester** A. Bazzano ${ }^{\mathbb{I l}}$, D.N. Burrows ${ }^{* *}$ and N. \\ Gehrels \\ *INAF-IASF Palermo, Via U. La Malfa 153, I-90146 Palermo, Italy \\ $\dagger$ INAF-IASF Milano, Via E. Bassini 15, I-20133 Milano, Italy \\ ** Department of Astronomy and Astrophysics, PSU, University Park, PA 16802, USA \\ ${ }^{\ddagger}$ NASA/Goddard Space Flight Center, Greenbelt, MD 20771, USA \\ ${ }^{\S}$ Universities Space Research Association, Columbia, MD, USA \\ IINAF-IASF Roma, Via Fosso del Cavaliere 100, I-00133, Roma, Italy
}

\begin{abstract}
We present the Swift observations of the supergiant fast X-ray transient (SFXT) SAX J1818.6-1703 collected during the most recent outburst, which occurred on May 62009. In particular, we present broad-band spectroscopic and timing analysis as well as a Swift/XRT light curve that spans more than two weeks of observations. The broad-band spectral models and length of the outburst resemble those of the prototype of the SFXT class, XTE J1739-302, further confirming SAX J1818.6-1703 as a member of this class.
\end{abstract}

Keywords: X-rays: binaries - X-rays: individual: SAX J1818.6-1703

PACS: 97.80.Jp, 98.70.Qy, 98.70.Rz

\section{RESULTS}

Here we summarize our main results on our campaign on SAX J1818.6-1703, obtained as a response of a Swift Cycle 5 GI trigger; further details are in Sidoli et al. [1].

We followed the evolution of the whole May 6 outburst of SAX J1818.6-1703 and its declining phase with $S$ wift/XRT. The source displays a dynamical range of more than 3000 (see Fig. 1, left) and a multiple-flaring behaviour as also observed in other SFXTs [2]. Temporally resolved spectroscopy of the XRT data did not reveal variability in the spectral parameters within the uncertainties, except for the change in the blackbody radius, when fitting the soft $\mathrm{X}$-rays with a single absorbed blackbody. We also did not find variability in the absorbing column density during the outburst.

We obtained, for the first time for this source, broad-band spectroscopy from soft to hard X-rays. Figure 1 (right) shows the Swift/XRT+BAT joint spectrum during the outburst obtained from strictly simultaneous data (only the blue XRT points in Fig. 1 left were considered). The broad-band spectrum can be characterized by either a very high absorption, a flat power law $(\Gamma \sim 0.1-0.5)$ with a cut-off at about $7-12 \mathrm{keV}$, or by Comptonized emission from a cold and optically thick corona, with an electron temperature $k T_{e}=5-7 \mathrm{keV}$, a hot seed photon temperature, $k T_{0}=1.3-1.4 \mathrm{keV}$, and an optical depth for the Comptonizing plasma $\tau=10$. The inferred $1-100 \mathrm{keV}$ luminosity is $3 \times 10^{36} \mathrm{erg} \mathrm{s}^{-1}$ (at $2.5 \mathrm{kpc}$; [3]). 

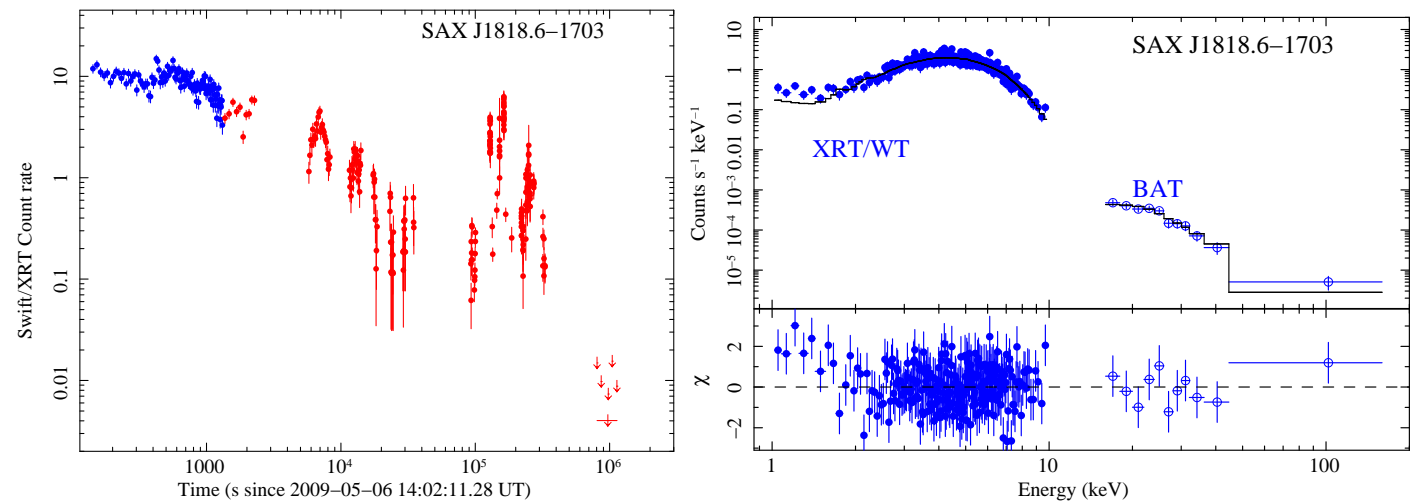

FIGURE 1. Left: Swift/XRT light curve, during the whole campaign (2009-05-06 to 2009-05-19). The four day gap before the $3 \sigma$ upper limits is due to the source being Moon constrained. The data shown were collected through both AT and GI ToO observations as well as regular ToO observations. The blue points were fitted simultaneously with BAT data. Right: Spectroscopy of the 2009 May 6 outburst. Top: simultaneous XRT/WT (filled circles) and BAT (empty circles) data fit with an absorbed Comptonization emission (bmc model in XSPEC). Bottom: the residuals of the fit (in units of standard deviations).

These broad-band properties are reminiscent of the X-ray spectral shape of the prototype of the SFXT class, XTE J1739-302 [4, 5]: similarly high absorption, X-ray luminosities, energy cutoff values, and hot seed photon temperatures. These spectral parameters indicate the presence of a cold and optically thick corona.

The observed properties of the broad band spectrum as well as the outburst duration of about 5 days, are quite similar to the ones observed in other SFXTs we have been monitoring in the last year and a half with Swift [6, and references therein] and confirm the fact that SAX J1818.6-1703 belongs to the class of SFXTs.

\section{ACKNOWLEDGMENTS}

This work was supported by contract ASI/INAF I/088/06/0 and I/023/05/0 in Italy, by NASA contract NAS5-00136 at PSU.

\section{REFERENCES}

1. L. Sidoli, P. Romano, P. Esposito, V. L. Parola, J. A. Kennea, H. A. Krimm, M. M. Chester, A. Bazzano, D. N. Burrows, and N. Gehrels, MNRAS 400, 258-262 (2009).

2. P. Romano, L. Sidoli, G. Cusumano, P. A. Evans, L. Ducci, H. A. Krimm, S. Vercellone, K. L. Page, A. P. Beardmore, D. N. Burrows, J. A. Kennea, N. Gehrels, V. La Parola, and V. Mangano, MNRAS 392, 45-51 (2009).

3. I. Negueruela, and M. P. E. Schurch, $A \& A$ 461, 631-639 (2007).

4. L. Sidoli, P. Romano, V. Mangano, G. Cusumano, S. Vercellone, J. A. Kennea, A. Paizis, H. A. Krimm, D. N. Burrows, and N. Gehrels, ApJ 690, 120-127 (2009).

5. L. Sidoli, P. Romano, L. Ducci, G. Cusumano, V. Mangano, A. Paizis, H. A. Krimm, S. Vercellone, D. N. Burrows, J. A. Kennea, and N. Gehrels, MNRAS 397, 1528-1538 (2009).

6. P. Romano, L. Sidoli, G. Cusumano, V. La Parola, S. Vercellone, C. Pagani, L. Ducci, V. Mangano, J. Cummings, H. A. Krimm, C. Guidorzi, J. A. Kennea, E. A. Hoversten, D. N. Burrows, and N. Gehrels, MNRAS 399, 2021-2032 (2009). 